\title{
Dealing with Non-Uniform Demands in Flexible GEO Satellites: The Carrier Aggregation Perspective
}

\author{
Eva Lagunas, Mirza Golam Kibria, Hayder Al-Hraishawi, Nicola Maturo and Symeon Chatzinotas \\ SnT - University of Luxembourg, Luxembourg \\ Corresponding Author: eva.lagunas@uni.lu
}

\begin{abstract}
To handle the increased broadband demand, commercial satellite operators are investing in advanced technology and intelligent solutions featuring smart payloads paving the way for satellites with a higher level of flexibility to efficiently support peaks in demand. This paper focuses on the optimization of the limited satellite frequency resources by considering the emerging Carrier Aggregation (CA) technique. Focusing on a multibeam multi-carrier GEO satellite system, we propose a CA design targeting a fair user-demand satisfaction across the system and we study two potential use-cases, namely the intra-beam CA (aggregation of carriers within a beam) and the inter-beam $\mathrm{CA}$ (aggregation of carriers across beams). The performance analysis demonstrates the benefits of $\mathrm{CA}$ in enhancing the peak data rate of satellite users as well as in improving the overall satellite resource utilization.
\end{abstract}

Index Terms-Carrier Aggregation, Heterogeneous Demand, Unmet and Unused Capacity

\section{INTRODUCTION}

Carrier Aggregation (CA), that is the association of multiple carriers to/from a single user terminal, was introduced in release 11 of $3 \mathrm{GPP}$ and is a key enabling technology in terrestrial radio communications networks e.g. Long Term Evolution (LTE) Advanced [1]. CA has become an essential element used in cellular wireless communications, enabling much higher data rates to those users experiencing peak data demands. The main idea behind CA is to exploit underutilized frequency resources to support users who are assigned to component carriers suffering from traffic congestion. Doing so, the high demand user can be satisfied while the overall system resource utilization is improved.

Given the benefits observed in the terrestrial wireless environment, the attention for $\mathrm{CA}$ grows steadily also for the satellite communications community. The application of the CA technique to satellite communications have been investigated within the context of the ESA funded activity CADSAT [2], where the benefits and drawbacks of CA have been analyzed through the development of a Software (SW)-based CA Demonstrator laboratory testbed.

With the user expectations for interactive data services becoming increasingly demanding and the inherent satellite spectrum scarcity, an intelligent allocation of frequency resources and the efficient usage of satellite transponders is mandatory [3]. The latter is becoming more relevant now with the non-uniform geographical distribution of the broadband traffic demand [4], [5]. Within the studies conducted in [2], CA has shown great promise in providing an additional degree of freedom on top of the conventional power and frequency assignment [6].

While previous radio resource management works such as [7]-[9] have targeted a demand-matching design at long term, CA should be seen as a technique to provide shortterm response to sudden peak data user demands on the system. In this paper, we focus on a multibeam multi-carrier GEO satellite system, where CA can take place (i) across carriers of the same beam and (ii) across carriers of adjacent beams. In particular, we present the CA design targeting a fair user-demand satisfaction across the system, which was already introduced in previous authors' work [6], [10]. The main contribution of this paper resides on the novel numerical results obtained with the CADSAT SW-Demo developed in [2]. In particular, we demonstrate that, in situations where sudden peak data rate occur and we cannot afford a complete reshuffling of the user-carrier assignment, CA shows significant benefits by simply aggregating carriers of the same beam in situations where we have congested and underutilized carriers and/or beams.

The rest of this paper is organized as follows. Section II introduces the GEO multibeam multi-carrier satellite system model. Section III introduces the proposed CA scheme, its optimization framework and the proposed solution. Supporting simulation results are presented in Section IV, and finally, concluding remarks are provided in Section V.

\section{System Model}

Consider the forward link of a bent-pipe GEO multi-beam satellite system with $N$ beams and a total of $U$ user terminals which are assumed to be distributed over the coverage area. Note that the users may vary among the beams. Different geographical distributions will be considered in the numerical results to emulate congested beams. The different user demands are defined as $d_{u}, u=1, \ldots, U$. The system has $K$ number of carrier components, each of bandwidth $B$.

Let us define the two assignment variables considered in this paper. First, let us define the user-carrier assignment matrix as A of dimensions $(K \times U)$, whose components $a_{k, u} \in\{0,1\}$ indicate if a user $u$ operates on carrier $k$. Since several users can be assigned to one carrier, we need to define the carrier fillrate $f_{k, u}$, which take a value between $0 \leq f_{k, u} \leq 1$ indicating the percentage of carrier $k$ 's bandwidth being assigned to user $u$. 
The data rate achievable by user $u$ operating in the complete bandwidth of carrier $k$ is defined as,

$$
r_{k, u}=B \cdot f_{\mathrm{SE}}\left(\mathrm{CINR}_{k, u}\right)
$$

where $f_{\mathrm{SE}}(\cdot)$ represents the function mapping the DVB-S2X spectral efficiency [11] associated with the corresponding Carrier-to-Interference and Noise Ratio (CINR) value. The expression of the CINR is provided in (2) below, where $p_{k}$ represents the transmit power.

$$
\mathrm{CINR}_{k, u}=\frac{p_{k}\left|h_{k, u}\right|^{2}}{\sigma^{2}}
$$

The channel coefficients $h_{k, u}$ are defined as

$$
h_{k, u}=\frac{\sqrt{G_{R} G_{u}[n]}}{4 \pi \frac{\ell_{u}}{\lambda}},
$$

where $G_{R}$ is the user terminal antenna gain, $G_{u}[n]$ denotes the gain from the $n$th satellite feed towards the $u$ th user and $\ell_{u}$ is the slant range between the satellite and the user $u$. Clearly, the computation of (1) requires knowledge of the channel-state information as well as link budget details.

Finally, the overall supplied capacity per user can be calculated as,

$$
s_{u}=\sum_{k=1}^{K} f_{k, u} \cdot a_{k, u} \cdot r_{k, u}
$$

In the next section, we formulate the $\mathrm{CA}$ design problem targeting a fair user-demand matching, meaning $d_{u} \approx s_{u}$, for the overal users in the system.

\section{USER-CARRIER ASSIGNMENT}

This section discusses the design of the user-carrier assignment variables $a_{k, u}$ and $f_{k, u}$, for $k=1, \ldots, K, u=1, \ldots, U$. We present an optimal and fair strategy, by formulating our optimization problem using one of the most popular fair allocation policies known as max-min fairness [12]. The optimization problem is presented in (5), where the utility function is based on the ratios $\left(s_{u} / d_{u}\right)$, which ideally should end up all being close or equal to 1 .

$$
\begin{array}{ll}
\underset{a_{k, u}, f_{k, u}}{\max } \min & \frac{s_{u}}{d_{u}} \\
\text { subject to } & \sum_{u=1}^{U} f_{k, u} \leq 1, \forall_{k}, \\
& \sum_{k=1}^{K} a_{k, u} \leq \Delta_{\max }, \forall_{u}, \\
& a_{k, u} \in\{0,1\}, \forall_{k}, \forall_{u}, \\
& 0 \leq f_{k, u} \leq 1, \forall_{k}, \forall_{u},
\end{array}
$$

The problem in (5) includes 4 different constraints. Constraint (5b) ensures that the resulting carrier assignment does not exceed the current carrier bandwidth. In other words, $\sum_{u=1}^{U} f_{k, u} B \leq B$. Constraint (5c) limits the total number of
TABLE I

Simulation PARAMETERS

\begin{tabular}{|c|c|}
\hline Parameter & Value \\
\hline Satellite location & GEO 13 E \\
Satellite Beam Pattern & From ESA CADSAT [2] \\
Max. Beam Gain $G_{u}[n]$ & $55 \mathrm{dBi}$ \\
User antenna gain $G_{R}$ & $42.1 \mathrm{dBi}(90 \mathrm{~cm}$ dish) \\
Downlink Frequency & $19.5 \mathrm{GHz}$ \\
Transmit Power per Beam & $12.24 \mathrm{~W}$ \\
Carrier Bandwidth & $54 \mathrm{MHz}$ \\
Channel & LoS channel (path loss) \\
\hline
\end{tabular}

carriers that can be aggregated by a particular user terminal to $\Delta_{\max }$. Given the current user terminal technology, there exists chips allowing to lock-in simultaneously into multiple carriers. However, these are usually limited to be not greater than 4. Finally, constraint (5d) enforces the binary behavior of the assignment parameter $a_{k, u}$, and constraint (5e) enforces the values of $f_{k, u}$ to be between 0 and 1 .

Problem (5) can be addressed by considering a slack variable $\psi$, such that $s_{u} \geq d_{u} \cdot \psi$. The resulting problem is stated in (6).

$$
\begin{array}{ll}
\underset{\begin{array}{l}
\max , f_{k, u} \\
a_{k, u}
\end{array}}{\text { subject to }} & \psi \\
& (5 \mathrm{~b}),(5 \mathrm{c}),(5 \mathrm{~d}),(5 \mathrm{e}), \\
& s_{u} \geq \psi \cdot d_{u}, \forall_{u},
\end{array}
$$

The optimization problem in (6) is a mixed-integer linear programming problem and can be efficiently solved with advanced optimization toolboxes like CVX [13].

\section{Simulation Results}

We now demonstrate the benefits of our proposed CA scheme in a multibeam multicarrier GEO satellite system. We divide this section into two practical use-cases: (i) Intra-beam CA (within carriers of the same beam), and (ii) Inter-beam CA (within carriers of different adjacent beams).

For the sake of simplicity, it is assumed that the users belong either to the high-demand type of users, whose average demand over time is $\tilde{d}_{h}$, or to the low-demand type of users, whose average demand over time is $\tilde{d}_{\ell}$. For this study, we take $\tilde{d}_{h}=20 \mathrm{Mbps}$ and $\tilde{d}_{\ell}=1 \mathrm{Mbps}$.

To assess the results, we will evaluate the unmet and unused capacity, which are defined as the total amount of demanded capacity that cannot be satisfied and the supplied capacity which exceeds the actual demand, respectively. In particular, we make use of the following expressions,

$$
\begin{aligned}
C_{u, \text { unmet }} & =\left(d_{u}-s_{u}\right)^{+}, \\
C_{u, \text { unused }} & =\left(s_{u}-d_{u}\right)^{+},
\end{aligned}
$$

where $(x)^{+}=\max (0, x)$.

The main simulation parameters are given in Table I. A 71-beam single-feed per-beam GEO satellite beam pattern provided by ESA is considered. 


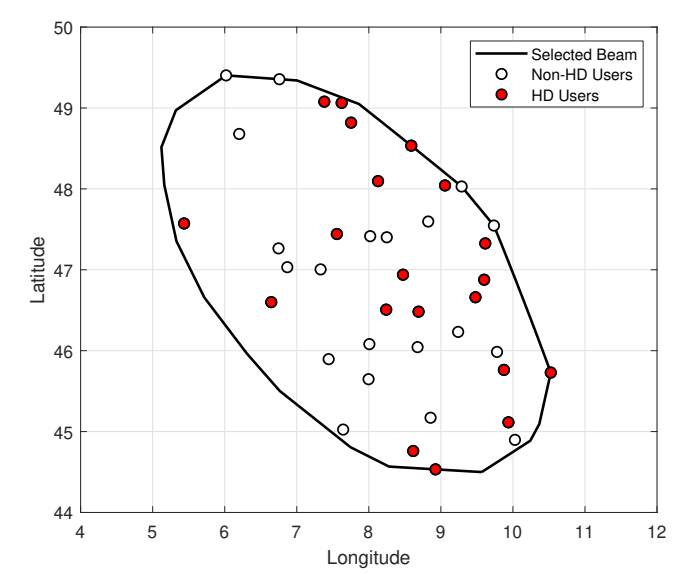

Fig. 1. Intra-Beam CA Scenario

\section{A. Intra-beam $C A$}

We consider a single-beam serving $U=40$ users with $K=$ 2 carriers. Users' locations are randomly distributed within the beam coverage area, as depicted in Fig. 1, where the highdemand users are highlighted in red. In particular, Step 1 is modeled assuming that half of the users, i.e. 20, are highdemand users.

To evaluate the benefits of $\mathrm{CA}$, we emulate the following situations:

- Step 1: Situation where the conventional non-CA system is doing fine in satisfying the demand of the system.

- Step 2: Situation where the conventional non-CA experience congestion in some carriers while some other carriers of the beam have spare capacity. By allowing CA, we expect to be able to satisfy the demand of user allocated to the congested carrier by aggregating with under-utilized carrier(s).

Assuming a system without CA but with a proper optimization of the user-carrier assignment, we achieve the allocation and demand-matching metrics presented in Table II. It can be observed that the system is very well balanced since the carrier supplied capacity is closely matched with the carrier demand. At a beam level, we obtain zero unused capacity, i.e. $\sum_{u=1}^{U} C_{u \text {,unused }}=0$, and minimal unmet capacity, i.e. $\sum_{u=1}^{U} C_{u \text {,unmet }}=4.94 \mathrm{Mbps}$. The satisfaction rates included in Table II are calculated as the percentage of demand being satisfy at carrier level. The achieved satisfaction rates in Step 1 are $100 \%$, which is close to the ideal situation of zero unmet capacity. Clearly, a top-to-bottom user-carrier assignment design without considering the CA capabilities seems to be enough to satisfy the demand of the beam.

Next, let us emulate a sudden carrier imbalance scenario, where one of the carriers struggles to satisfy the associated users, while the other carrier has spare bandwidth. This is emulated by taking the same user distribution as in Step 1 and incrementing the number of HD-Users in one carrier while reducing the number of HD-Users in other carriers, always keeping the total number of users fixed to $U=40$. The
TABLE II

USER-CARrier AsSignMent IN SteP 1 (W/O CA)

\begin{tabular}{|c|c|c|}
\hline & Carrier 1 & Carrier 2 \\
\hline Number of High-Demand Users & 9 & 11 \\
\hline Number of Low-Demand Users & 15 & 5 \\
\hline Demand per Carrier & $198.14 \mathrm{Mbps}$ & $202.20 \mathrm{Mbps}$ \\
\hline Supplied Capacity per Carrier & $195.70 \mathrm{Mbps}$ & $199.71 \mathrm{Mbps}$ \\
\hline Satisfaction Rate & $98.78 \%$ & $98.77 \%$ \\
\hline
\end{tabular}

TABLE III

USER-CARRIER ASSIGNMENT IN STEP 2

\begin{tabular}{|c|c|c|}
\hline & Carrier 1 & Carrier 2 \\
\hline Number of High-Demand Users & 14 & 5 \\
\hline Number of Low-Demand Users & 10 & 11 \\
\hline Demand per Carrier & $288.31 \mathrm{Mbps}$ & $97.47 \mathrm{Mbps}$ \\
\hline Supplied Capacity per Carrier (w/o CA) & $195.70 \mathrm{Mbps}$ & $199.71 \mathrm{Mbps}$ \\
\hline Satisfaction Rate (w/o CA) & $67.88 \%$ & $100 \%$ \\
\hline Supplied Capacity per Carrier (w/ CA) & $295.75 \mathrm{Mbps}$ & $99.98 \mathrm{Mbps}$ \\
\hline Satisfaction Rate (w/ CA) & $100 \%$ & $100 \%$ \\
\hline
\end{tabular}

congestion of the system becomes evident from the results without the application of CA shown in Table III, where the demand of carrier 1 (288.31 Mbps) is much higher than the supplied capacity (195.70 Mbps). On the other hand, we can observe that carrier 2 has spare capacity, as the demand is much lower than the supplied capacity. In this case where a system without CA capabilities is assumed, we obtain a system unused capacity of $\sum_{u=1}^{U} C_{u \text {, unused }}=125.54 \mathrm{Mbps}$ (mostly associated to the underloaded carrier), and a system unmet capacity of $\sum_{u=1}^{U} C_{u \text {, unmet }}=115.92 \mathrm{Mbps}$ (mostly associated to the overloaded carrier).

To overcome the congestion caused by sudden demand changes, we consider now a system with CA capabilities. As shown in the last two rows of Table III, a system with CA capabilities is able to completely satisfy the demand in carrier 1 by exploiting the unused resources of carrier 2 . This is achieved by simply allowing $5 \mathrm{HD}$-users of carrier 1 to aggregate with carrier 2. In Fig. 2, we compare the unmet and unused capacity per carrier level with and without CA for the particular example emulated in this section. It can be seen that both unmet and unused capacity are kept to minimal values when considering $\mathrm{CA}$, thus improving the satellite resource utilization. In particular, the system unmet capacity is $\sum_{u=1}^{U} C_{u, \text { unmet }}=0 \mathrm{Mbps}$, and the system unused capacity is $\sum_{u=1}^{U} C_{u \text {, unused }}=9.95 \mathrm{Mbps}$,

In conclusion, for sudden variations of the user demands, the intra-beam CA is shown to achieve higher demand satisfaction rates without the need to reshuffle the whole user-carrier assignment by simply exploiting underutilized resources of other carriers of the system.

\section{B. Inter-beam $C A$}

For this section, we consider $N=2$ adjacent beams serving $U=20$ users with $K=2$ carriers (one carrier per beam). The selected beams and an example of users' locations distribution are depicted in Fig. 3, where the high-demand users are highlighted in red. The scenario shown in Fig. 3 considers 


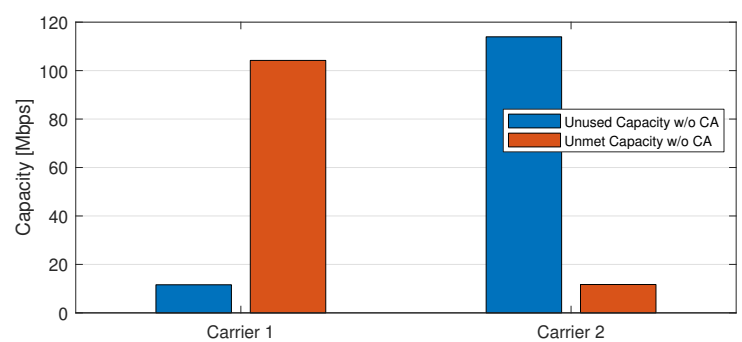

(a)

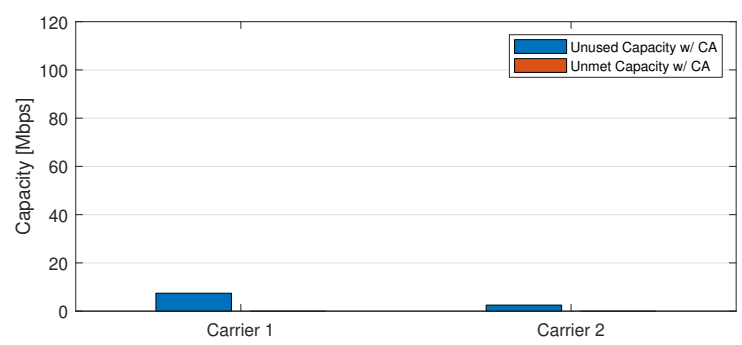

(b)

Fig. 2. Unmet and unused carrier capacity: (a) w/o CA; (b) w/ CA

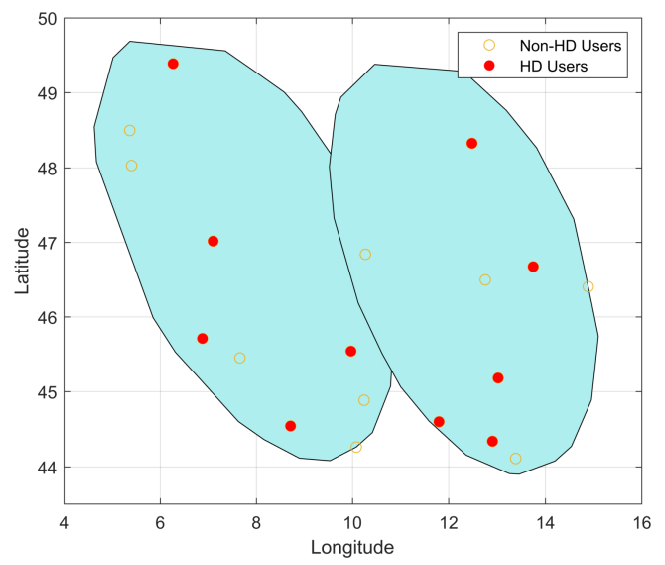

Fig. 3. Inter-Beam CA Scenario with a split of $50 \%-50 \%$ of users on each beam

a split of $50 \%-50 \%$ of users on each beam. To emulate the hot-cold beam scenario, we will tune these percentages towards $70 \%-30 \%$ and an extreme case of $90 \%-10 \%$.

Table IV presents the results in terms of system unmet and unused capacity for the 2 beam single-carrier system under consideration. The results have been obtained by averaging a total of 20 realizations (by randomly varying the user demands around the mean values $\tilde{d}_{h}$ and $\left.\tilde{d}_{\ell}\right)$. From Table IV, we can extract the following conclusions:

- The system w/o CA is designed such that each beam is able to satisfy approximately half of the users of the system. For this reason, when we test the $50 \%-50 \%$ scenario, it achieves the lowest unmet and unused capacity. Note that the unmet capacity is always equal to the unused capacity for the system w/o CA system because of the same reason.

- $\mathrm{CA}$ is able to outperform the system w/o CA in terms
TABLE IV

SYSTEM UNMET AND UNUSED CAPACITY [MBPS] FOR THE HOT-COLD BEAM SCENARIO

\begin{tabular}{|c|c|c|c|c|}
\hline & & $50 \%-50 \%$ & $70 \%-30 \%$ & $90 \%-10 \%$ \\
\hline \multirow{2}{*}{ w/o CA } & Unmet & 19.64 & 177.16 & 312.28 \\
& Unused & 19.64 & 177.16 & 312.28 \\
\hline \multirow{2}{*}{ w/ CA } & Unmet & 4.12 & 21.48 & 81.11 \\
& Unused & 0 & 0 & 0 \\
\hline
\end{tabular}
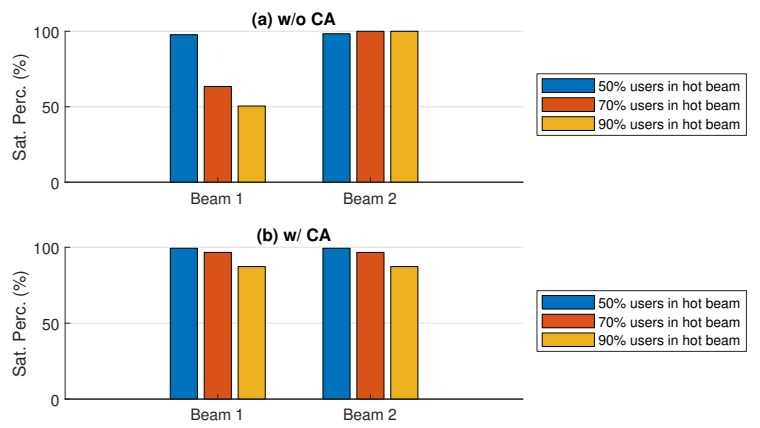

Fig. 4. Inter-Beam Demand Satisfaction Rate Results

of unused and unmet capacity in all scenarios. It can be observed that CA exploits all the resources of the system (i.e. unused $0 \mathrm{Mbps}$ ) to reduce the unmet capacity as much as possible.

Fig. 4 shows the average beam demand satisfaction rate for the same realizations as in Table IV. We can observe that the system without CA is struggling to satisfy the demand of beam 1 (i.e. hot beam), while CA is providing always higher demand satisfaction percentages.

Finally, Fig. 5 and Fig. 6 shows the per-beam unmet and unused capacity, prespectively, for the system considering noCA (top-subfigure) and CA (bottom-subfigure). We can see how the system without CA experiences unmet capacity in the hot beam (i.e. beam 1) while it has a significant amount of unused capacity in the cold beam (i.e. beam 2). Interestingly, the CA system is able to share resources across beams and achieves a minimum unmet and unused capacity.

\section{CONCLUSIONS AND REMARKS}

This paper analyzes the benefits of the CA technique applied to satellite communications systems. We proposed an efficient carrier assignment optimization framework for which we run extensive numerical simulations targeting two main application use-cases, namely the intra-beam CA and the inter-beam CA. For sudden variations of the user demands, the intra-beam $\mathrm{CA}$ was shown to achieve higher demand satisfaction rates without the need to reshuffle the whole user-carrier assignment by simply exploiting underutilized resources of other carriers of the system. Similarly, the evaluation of the applicability of CA in the hot-cold beam scenario evidence the benefits of $\mathrm{CA}$ in exploiting the extra/unused resources of the neighboring cold-beams to enhance the offered capacity at the congested or hot-beams. 


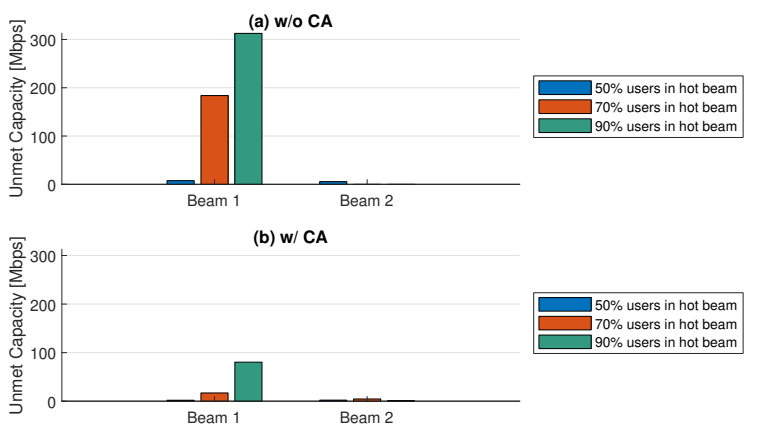

Fig. 5. Per-Beam Unmet Capacity Results

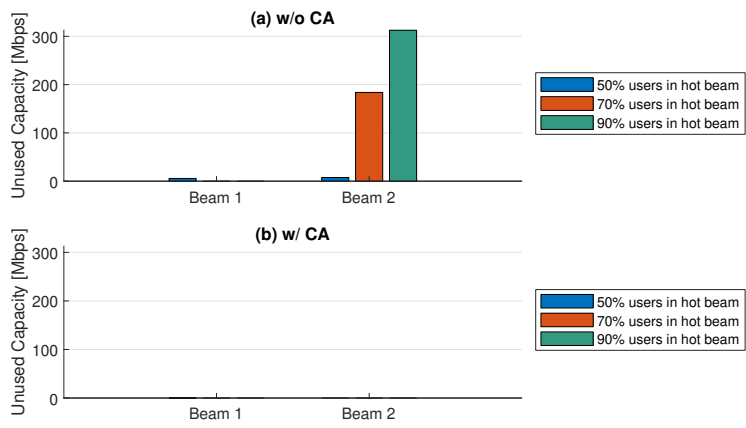

Fig. 6. Per-Beam Unused Capacity Results

It shall be noted that success of the deployment of CA in satellite communications systems entail advance packet scheduling schemes at the gateway side. For further information on this aspect, the reader is referred to [14] for details on how to introduce CA into satellite systems from a link layer perspective.

\section{ACKNOWLEDGMENT}

This work has been partially supported by the Luxembourg National Research Fund (FNR) under the project FlexSAT "Resource Optimization for Next Generation of Flexible SATellite Payloads" (C19/IS/13696663) and by the European Space Agency (ESA) funded activity CADSAT: Carrier Aggregation in Satellite Communication Networks. Please note that the views of the authors of this paper do not necessarily reflect the views of ESA.
The author would like to thank J. Grotz and S. Andrenacci from SES, Luxembourg, for their helpful advice on various practical aspects related to the application of CA on satellite systems. The authors would also like to thank N. Toptsidis, technical officer managing the CADSAT project, for his insightful comments on the technical aspects of this paper.

\section{REFERENCES}

[1] A. Roessler and M. Kottkamp, "White Paper: LTE-Advanced (3GPP Rel.11) Technology Introduction," https://www.rohde-schwarz.com/, 2014.

[2] ESA CADSAT Project: Carrier Aggregation in Satellite Communication Networks. [Online]. Available: https://artes.esa.int/projects/cadsat

[3] "SES and Thales Unveil Next Generation Capabilities On-Board SES-17, April 2017," https://www.ses.com/press-release/ses-and-thalesunveil-next-generation-capabilities-onboard-ses-17, accessed: 2020-02.

[4] V. Icolari, S. Cioni, P. Arapoglou, A. Ginesi, and A. Vanelli-Coralli, "Flexible precoding for mobile satellite system hot spots," in 2017 IEEE International Conference on Communications (ICC), 2017, pp. 1-6.

[5] O. Kodheli et al., "Satellite Communications in the New Space Era: A Survey and Future Challenges," Feb. 2020, arXiv:2002.08811.

[6] M. Kibria, E. Lagunas, N. Maturo, D. Spano, H. Al-Hraishawi, and S. Chatzinotas, "Carrier Aggregation in Multi-Beam Satellite Communications," IEEE GlobeCom, Hawaii, USA, Dec. 2019.

[7] A. Freedman, D. Rainish, and Y. Gat, "Beam Hopping - How To Make it Possible," Ka and Broadband Communication Conference, Bologna, Italy, Oct. 2015.

[8] A. I. Aravanis, B. Shankar M. R., P. Arapoglou, G. Danoy, P. G. Cottis, and B. Ottersten, "Power allocation in multibeam satellite systems: A two-stage multi-objective optimization," IEEE Transactions on Wireless Communications, vol. 14, no. 6, pp. 3171-3182, June 2015.

[9] G. Cocco, T. de Cola, M. Angelone, Z. Katona, and S. Erl, "Radio resource management optimization of flexible satellite payloads for dvbs2 systems," IEEE Transactions on Broadcasting, vol. 64, no. 2, pp. 266-280, June 2018

[10] M. Kibria, E. Lagunas, N. Maturo, H. Al-Hraishawi, and S. Chatzinotas, "Carrier Aggregation in Satellite Communications: Impact and Performance Study," IEEE Open Journal of the Communications Society, under revision.

[11] "Digital Video Broadcasting (DVB). (2014). DVB-S2X Standard [Online]," https://www.dvb.org/standards/dvb-s2x, accessed: 2020-06.

[12] B. Radunovic and J. Le Boudec, "A unified framework for max-min and min-max fairness with applications," IEEE/ACM Transactions on Networking, vol. 15, no. 5, pp. 1073-1083, 2007.

[13] M. Grant and S. Boyd, "CVX: Matlab software for disciplined convex programming, version 2.1," http://cvxr.com/cvx, Mar. 2014.

[14] H. Al-Hraishawi, N. Maturo, E. Lagunas, and S. Chatzinotas, "Perceptive Packet Scheduling for Carrier Aggregation in Satellite Communication Systems," IEEE International Conference on Communications (ICC), Jun. 2020. 